\title{
Periodic Vehicle Routing problem and tabu search algorithm
}

\author{
Wen Wenhua \\ Transportation Management College \\ Dalian Maritime University \\ Dalian, China \\ wwhuasz@163.com \\ Fan Houming \\ Transportation Management College \\ Dalian Maritime University \\ Dalian, China \\ fhm468@163.com
}

\author{
Yang Xiang \\ Transportation Management College \\ Dalian Maritime University \\ Dalian, China \\ yx_420@sohu.com \\ Qi Tongxuan \\ Transportation Management College \\ Dalian Maritime University \\ Dalian, China \\ qtx0620@yahoo.cn
}

\begin{abstract}
Periodic vehicle routing problem and its solution algorithms are being deeply studied by scholars, the outputs of them are helpful to solve problems such as time setting for waste household appliances, bulky waste discarded in reality, etc. Through the literatures review of PVRP, it can be seen that PVRP solution is still difficult. Taking the reverse recovery system as an example and aiming at the shortest distance, this paper builds the periodic vehicle routing optimization model which can fully embody the characteristics and requirement of recycling of waste household appliances, designs an improved tabu search algorithm, and develops a program by Matlab to solve the established model. Example analysis shows the validity of the model and algorithm. This work can enrich related theory of PVRP and reverse logistics, and provide reference for government to make decisions of relevant management policies.
\end{abstract}

Keywords- periodic vehicle routing problem; vehicle routing problem;optimizing; tabu search; algorithm desigining

\section{INTRODUCTION}

PVRP(Periodical Vehicle Routing Problem, VRP), which was originally put forward by Beltrami and Bodin in 1974 [1], is the important branch of VRP (Vehicle Routing Problem, VRP). VRP usually solves route optimization problem in a period of time such as a day, whereas PVRP expanded multi-periods such as $\mathrm{S}$ days to complete service for customers order and to meet the needs of them accordingly.

Existing in reality, there are problems such as time setting for waste household appliances, bulky waste discarded. If recycling vehicle can be scheduled periodically, can the harm to environment and the cost of recycling be reduced obviously. Therefore, study on the PVRP has important theoretical and practical significance.

Since PVRP problem was put forward, scholars have been studying on the solving method for it. Russell and Igo (1979) [2] solved the PVRP by using an optimization heuristic algorithm to get the initial solution at first, and then building a heuristic algorithm. Christofides and Beasley (1984) [3] proposed the points assigned to a specific time and use the two phase exchange optimization algorithms; Tan and Beasley (1984) [4] designed heuristic algorithm which divided PVRP into two layers to solve the assignment problem by task matrix first, and then to determine the customer service time and solve VRP problems every day. Russell and Gribbin (1991) [5] used the method of approximate network flow model to build the initial route, and then used heuristic algorithm for the adjusted optimization of route. Chao (1995) [6] slacked vehicle capacity constraints to obtain initial solution first, and then to improve by point adjustment. Cordeau (1997) [7] based on the framework of tabu search, designed the special insert and route optimization method to solve the PVRP, and set the objective function for the total distance and penalty of beyond the capacity; Drummond (2001) [8] designed a combination of genetic algorithm and the neighborhood search algorithm to solve the PVRP, which regarded chromosomes as two vectors, one for PVRP cycles and the feasible time combination of corresponding points, and the other for the accumulation of daily demand. The total distance of PVRP was taken as Fitness function, and a set of standard solutions was gotten from the crossover and mutation operations. Hemmelmayr (2009) [9] designed variable neighborhood search (VNS), with a randomly assigned way, made customer clustering and built route with saving algorithm, and then used VNS to solve the PVRP and PTSP problem. The results showed that VNS was a better algorithm in the relatively large size calculation; Bin $\mathrm{Yu}$ (2011) [10] proposed an improved ant colony optimization algorithm to solve PVRP problem with time Windows, and used the multidimensional pheromone matrix and multi-point crossover operation to improve the performance of the algorithm; Cordeau (2012) [11] proposed a parallel iterative tabu search algorithm to solve the four VRPs including PVRP route optimization problem, which based on the global search and tabu search as simple interference mechanism to ensure the search range broadly.

Through the literature review of PVRP, it can be seen that PVRP solution algorithm design is difficult. Early heuristic algorithm generally solved the assignment 
problem and then to solve the routing problem, of which advantage is easy to understand and the amount of calculation is relatively small. But the optimization results are not very good. Recent studies have focused on the meta-heuristic algorithm[12], which solve the problem of assignment and the route at the same time. The metaheuristic algorithm optimization effect is ideal, but more complex. So this article adopted the tabu search algorithm to solve waste household appliances recycling PVRPs based on the analysis above.

\section{PROBLEM DESCRIPTION AND MODEL BUILDING}

\section{A. Problem description}

Waste household appliances recycling network vehicle routing problem can be described as: inside the region, a collection of waste household appliances centers cover several collection points, residents send waste household appliances to the points on schedule, and collection center is responsible for recycling them from the points regularly. In planned period, recovery vehicles start from a collection center every day to the points scheduled, and return to the collection center with the waste household appliances collected finally. All collection points inside the region should be serviced during the period $\mathrm{S}$ days (assuming for 7 days).

\section{B. $\quad$ model building}

PVRP general form is: given A complete graph $\mathrm{G}=(\mathrm{N}$, A), $\mathrm{N}$ point set for the customer, $\forall i, j \in N, a_{i j}$ is the arc to connect the customer points $i$ and customer $j$, the cost of the $\operatorname{arc} d_{i j}$ is fixed and known, only depot $i=0$, collection points for $\mathrm{Nc}=\mathrm{N} /\{0\}$, vehicles for $\mathrm{K}$, capacity limits for C. For Period S days program, $\forall i \in N_{c}$, the total demands of all collection points for $\mathrm{Wi}$, the number of service time needed for $f_{i}$, the service time required for $\mathrm{T}_{\mathrm{i}}$. The solving objective is to minimize the total transportation cost for meeting the demand of all collection points in the period.

Model parameters: set all of the time required for $\mathrm{S}$, $s \in S$ represents a time requirement, any one time requirement in $\mathrm{S}$ can be represented by a vector $a_{s t}$ :

$$
\begin{gathered}
a_{s t}=\left\{\begin{array}{l}
1, \text { if } t \text { equals to or less than any time required } s, s \in S, t \in T ; \\
0, \text { else }
\end{array}\right. \\
S_{i}=\left\{s \in S: \sum_{t \in T} a_{s t}=f_{i}\right\} \text { refers to alternative time set }
\end{gathered}
$$
required for any customer point ( $\forall i \in N_{c}$ ).

$\omega_{i}=W_{i} / f_{i}$, refers to service quantity each time, which is equal to total demand Wi divided by number of service time $f_{i}$ needed.

Model decision variables: $x_{i j k}^{t}=\left\{\begin{array}{l}1, \text { if the vehicle } k \in K \text { passes through } \operatorname{arc}(i, j) \in \text { A on the day } t \in T ; \\ 0, \text { else }\end{array}\right.$ $z_{i}^{s}=\left\{\begin{array}{l}1, \text { if selecting a required time } s \in S \text { to service any defined customer point } i \in N_{c} \\ 0, \text { else }\end{array}\right.$ $v_{i}^{t}=\left\{\begin{array}{l}1, \text { if the customer point } \mathrm{i} \in N_{c} \text { is serviced on the day } t \\ 0, \text { else }\end{array}\right.$
With the objective of the shortest distance, PVRP model was built as follow:

$$
\begin{aligned}
& \min \sum_{\mathrm{t} \in T} \sum_{k \in K} \sum_{i \in N} \sum_{j \in N} x_{i j k}^{t} d_{i j} \\
& \text { s.t. } \\
& \sum_{s \in S_{i}} z_{i}^{s}=1, \forall i \in N_{c} \\
& v_{i}^{t}=\sum_{s \in S_{i}} z_{i}^{s} a_{s t} \quad \forall \boldsymbol{t} \in \boldsymbol{T}, \boldsymbol{i}, \boldsymbol{j} \in N_{c} \\
& \sum_{k \in K} x_{i j k}^{t} \leq \frac{v_{i}^{t}+v_{j}^{t}}{2} \quad \forall t \in T, i, j \in N_{c}, i \neq j \\
& \sum_{j \in N_{c}} x_{j i k}^{t}=\sum_{j \in N_{c}} x_{i j k}^{t} \quad \forall t \in T, i \in N, k \in K \\
& \sum_{k \in K} \sum_{i \in N} x_{i j k}^{t}=\left\{\begin{array}{l}
v_{j}^{t}, \forall t \in T, j \in N_{c} \\
|\mathrm{~K}|, \forall t \in T, j=0
\end{array}\right. \\
& \sum_{i \in Q} \sum_{j \in Q} x_{i j k}^{t} \leq|Q|-1, \forall t \in T, k \in K, Q \subseteq N_{c} \\
& \sum_{j \in N_{c}} x_{0 j k}^{t} \leq 1 \quad \forall t \in T, k \in K \\
& \sum_{i \in N_{c}} \omega_{i} \sum_{j \in N} x_{i j k}^{t} \leq C \quad \forall t \in T, k \in K \\
& \sum_{i \in N} \sum_{j \in N} x_{i j k}^{t} d_{i j} \leq L \quad \forall t \in T, k \in K \\
& z_{i}^{s} \in\{0,1\}, \forall i \in N_{c}, t \in T \\
& x_{i j k}^{t} \in\{0,1\}, \forall i \in N, j \in N, t \in T, k \in K
\end{aligned}
$$

Among all constraint conditions, (2) guarantees a feasible time required for each collection point; (3) guarantees service time required for any collection point within selected time required; (4) ensures that the two collection points serviced in sequence must be arranged on the same day; (5) reflects flow-balance, which means, to any point, vehicles inflow is equal to outflow on the day t; (6) means that all points in everyday plan have been serviced, none left; (7) eliminates constraints for sub-loop; (8) indicates once a day per car; (9) and (10) represents constraints of vehicle capacity and the longest distance accordingly; (11) and (12) define value range of variables.

\section{ALGORITHM DESIGN}

This paper adopted the improved Tabu Search to solve the PVRP model. Tabu Search (TS) is a meta-heuristics search technique. In order to evade the main shortcomings of local neighborhood search getting stuck in suboptimal regions or on plateaus where many solutions are equally fit, it uses tabu list to record the local optimal points reached and the next search will no more or explore these points selectively according to information on the list

This article aimed at the solving approach of PVRP

Step 1: Build initial solution s, let tabu list $\mathrm{H}=\Phi$;

Step 2: If it meets termination criterion, move to the third step, otherwise, select the best solution $\mathrm{s}^{\prime}$ in the neighborhood $\mathrm{N}(\mathrm{s})$ of $\mathrm{s}$, then let $\mathrm{s}=\mathrm{s}^{\prime}$, improve $\mathrm{H}$ and repeat Step 2;

Step 3: Output the results and stop. 


\section{A. Building of initial solution}

- Build a coordinate system, determine the position coordinates of collection center and points in the system.

- Select a service time combination $S_{i}$ for each collection point i randomly.

- Put $\mathrm{i}$ selected at random on the route $\mathrm{k}(\mathrm{k}=1,2, \ldots \ldots \mathrm{K})$ on the basis of distance from collection center for each day $\mathrm{t}$.

- If the total transport volume of the route $\mathrm{k}$ exceeds the loading instructions of the vehicle $\mathrm{k}$ after $\mathrm{i}$ has been involved on the day $t$, then let $k=k+1$, repeat the third step.

\section{B. Algorithm design}

The key to design of Tabu search includes searching method, neighborhood construction, tabu objectives, evaluation function, tabu length, determination of candidate set, amnesty rules and terminate criterion. The construction method of the algorithm is listed as below:

Searching method: Based on Tabu search, the improvement of initial solution is designed. Common searching algorithm such as Relocate, 2-opt, 1-exchange and cross heuristic are mostly used, while this paper adopted 2-opt.

Neighborhood construction: Set a group of attribute vectors $B(s)=(i, k, t)$, which mean collection point $i$ is serviced by the vehicle $\mathrm{k}$ on the day for every group of feasible solutions. The neighborhood of s, N(s), can be constructed in both ways as follows:

- Transfer the collection point $\mathrm{i}$ from the vehicle $\mathrm{k}$ to other vehicle $\mathrm{k}^{\prime}$ on the same day $\mathrm{t}$.

- Change present service time combination $S_{\mathrm{i}}$ for other feasible combinations on the basis of $s_{i}{ }^{\prime} \in S$; for $\mathrm{t}=1,2 \ldots \ldots \mathrm{T}$, if both $a_{s t}=1$ and $a_{s^{\prime} t}=0$, then eliminate customer point i from the transport route on the day $\mathrm{t}$; if both $a_{s t}=0$ and $a_{s^{\prime} t}=1$, then put $\mathrm{i}$ on the route.

Tabu objective: if the customer point $\mathrm{i}$ has been removed from the vehicle $\mathrm{k}$ on the day $\mathrm{t}$, then suppose that this point cannot be removed back in next several cycles, so put attribute vector $(\mathrm{i}, \mathrm{k}, \mathrm{t})$ on the tabu list, recorded as Tikt.

Evaluation function: take transport distance as evaluation function.

Tabu length: Tabu length is the iteration step number that tabu objectives are not allowed to be selected. Here it's a constant which is determined by scale of the problem.

Determination of candidate set: Choose the best tabu_length+1 element in the neighborhood as the determination of candidate set.

Amnesty rules: in order to not only improve the quality of solution but also avoid cycles, amnesty rules is introduced. It can be defined as follow: when the current optimal solution didn't decrease until the given value, the optimal solution on the tabu list would become the initial solution of the next iteration.

Terminate criterion: Given maximum iteration step number or amnesty number is exceeded.

\section{EXAMPLE ANALYSIS}

TABLE I. THE COORDINATES OF 33 COLLECTION POINTS COVERED BY THE CENTER AND ESTIMATED QUANTITY OF WASTE HOUSEHOLD ELECTRICAL APPLIANCES GENERATED PER WEEK

\begin{tabular}{|c|c|c|c|c|}
\hline community & $\begin{array}{c}\mathbf{X} \\
\text { axes }\end{array}$ & $\begin{array}{c}\mathbf{Y} \\
\text { axes }\end{array}$ & $\begin{array}{l}\text { average quantity } \\
\text { generated }(\mathrm{kg})\end{array}$ & $\begin{array}{c}\text { Service } \\
\text { frequency }\end{array}$ \\
\hline 1 & 33 & 37 & 671.04 & 1 \\
\hline 2 & 40 & 40 & 586.66 & 1 \\
\hline 3 & 14 & 34 & 746.22 & 1 \\
\hline 4 & 32 & 22 & 549.07 & 1 \\
\hline 5 & 23 & 27 & 682.16 & 1 \\
\hline 6 & 36 & 14 & 266.96 & 1 \\
\hline 7 & 90 & 37 & 741.81 & 1 \\
\hline 8 & 96 & 28 & 771.34 & 1 \\
\hline 9 & 80 & 47 & 765.01 & 1 \\
\hline 10 & 65 & 44 & 808.36 & 2 \\
\hline 11 & 46 & 61 & 1225.48 & 2 \\
\hline 12 & 38 & 60 & 430.36 & 1 \\
\hline 13 & 35 & 53 & 546.38 & 1 \\
\hline 14 & 52 & 58 & 614.08 & 1 \\
\hline 15 & 45 & 53 & 573.23 & 1 \\
\hline 16 & 47 & 53 & 438.41 & 1 \\
\hline 17 & 36 & 45 & 698.47 & 1 \\
\hline 18 & 66 & 62 & 421.15 & 1 \\
\hline 19 & 64 & 73 & 444.93 & 1 \\
\hline 20 & 62 & 66 & 532.58 & 1 \\
\hline 21 & 67 & 55 & 535.26 & 1 \\
\hline 22 & 73 & 72 & 450.68 & 1 \\
\hline 23 & 58 & 55 & 295.34 & 1 \\
\hline 24 & 62 & 75 & 2489.32 & 2 \\
\hline 25 & 55 & 70 & 942.79 & 1 \\
\hline 26 & 47 & 74 & 1130.36 & 2 \\
\hline 27 & 40 & 66 & 462.38 & 1 \\
\hline 28 & 30 & 83 & 353.64 & 1 \\
\hline 29 & 35 & 78 & 686.96 & 1 \\
\hline 30 & 45 & 83 & 484.82 & 1 \\
\hline 31 & 35 & 95 & 1047.89 & 2 \\
\hline 32 & 30 & 101 & 576.11 & 1 \\
\hline 33 & 38 & 90 & 480.99 & 1 \\
\hline \multicolumn{3}{|c|}{ total } & 21779.21 & \\
\hline
\end{tabular}

There are a large number of waste household electrical appliances generated in China every year, which will do great damage to the environment if they are not timely recovered. Many local governments have begun to centralized recovery them irregularly and made some achievements, but have not achieved the ideal effect. From 
the point of the current situation, setting the collection centers and collection point network, scheduling periodical recovery vehicle are the keys to solve the problem. Thus, this paper has practical significance to research optimization problems by using routes in waste household appliances recycling network system[13].

The coordinates of a collection center in the District of the City is $(50.7107,60.4552)$, covering 33 collection points. It can be estimated that the average number of waste household electrical appliances at every collection point per week. Supposed that for each i, whose weekly collected total quantity is more than $800 \mathrm{~kg}$, would be serviced twice a week and the interval time is 3 days, then service quantity each time would be half of total quantity. See table I .

Table I shows that the total transport volume a week is about 21.7 tons, averaging 3.1 tons per day. It is assumed that one vehicle can complete the transportation because of minor transport volume in this case. Depending on the algorithm listed above, Matlab programming is employed to solve the model. The results obtained are shown in table II.

TABLE II. OPTIMIZATION RESULTS

\begin{tabular}{|c|c|}
\hline Day t & vehicle routing orders \\
\hline 1 & collection center-23-26-30-22-3-collection center \\
\hline 2 & collection center-11-20-18-10-31-collection center \\
\hline 3 & collection center-25-13-7-8-collection center \\
\hline 4 & collection center-26-24-19-33-32-collection center \\
\hline 5 & collection center-16-15-12-27-5-4-6-collection center \\
\hline 6 & collection center-14-11-25-21-10-28-31-collection center \\
\hline 7 & collection center-24-29-17-2-1-collection center \\
\hline
\end{tabular}

As seen in Table II, the length of current optimal route is $1047.7 \mathrm{~km}$, the vehicle starting from collection center every day and providing service 7 days a week in order of: day $1-23-26-30-22-3$; day 2-11-20-18-10-31; day $3-25-13-7-8$; day 4-26-24-19-33-32; day 5-1615-12-27-5-4-6; day 6-—14-11-25-21-10-28-31; day 7-24-29-17-2-1.

The government, on this basis, can make policies such as the waste household appliances emissions policy of urban community residents, which only one day before the recycling vehicle the vehicle routing through them, so that the harm to environment and the cost of recycling are reduced.

\section{CONCLUSIONS}

This article built PVRP model of waste household appliances recycling network with the objectives of resource recycle and the lowest recycling lost, which can fully embody the features and requirements of waste household appliances recycling and satisfy its comprehensive profits. Based on the analysis of heuristic algorithm, TS is employed to solve PVRP model and improved about the characteristics of the model. The solution of the model was realized by Matlab program. An example analysis indicates that both the model and the algorithm are effective. This work can enrich the related theories of PVRP and reverse logistics, provides the reference for the city of the vehicle dispatching decisionmaking.

\section{ACKNOWLEDGMENT}

This work was supported by the National Natural Science Foundation of China (Grant No. 61473053), Liaoning Province Department of Education Science and technology research projects(Grant No. L2014196), and the Fundamental Research Funds for the Central Universities.

\section{REFERENCES}

[1] E. J. Beltrami, L. D. Bodin, Networks and vehicle routing for municipal waste collection[J]. Networks, 1974, 4 (1): 65-94.

[2] R. Russell, W. Igo. An Assignment Routing Problem[J]. Networks, 1979, 9: 1-17

[3] N. Christofides, J.E. Beasley. The period vehicle routing[J]. Networks, 1984, 14: 237-256.

[4] C.C.R. Tan, J.E. Beasley. A heuristic algorithm for the period vehicle routing problem[J]. Omega,1984,12(5):497-504.

[5] R. Russell, D. Gribbin, A multiphase approach to the period routing problem[J]. Networks, 1991, 21(7): 747-765.

[6] I.M. Chao, B. Golden, E. Wasil. An improved heuristic for the period vehicle routing problem[J]. Networks, 1995, 26(1): 25-44.

[7] J.F. Cordeau, M. Gendreau, G. Laporte. A tabu search heuristic for periodic and multi-depot vehicle routing problems[J]. Networks, 1997, 30(2):105-119.

[8] Lúcia M.A. Drummond, Luiz S. Ochi, Dalessandro S. Vianna. An asynchronous parallel metaheuristic for the period vehicle routing problem[J]. Future Generation Computer Systems, 2001, 17(4): 379-386.

[9] Hemmelmayr V. C., Doerner K. F., Hartl R. F. A variable neighborhood search heuristic for periodic routing problems[J]. European Journal of Operational Research, 2009, 195(3): 791-802.

[10] B. Yu, Z. Z Yang. An ant colony optimization model: The period vehicle routing problem with time windows $[\mathrm{J}]$. Transportation Research Part E, 2011, 47(2):166-181.

[11] J.F. Cordeau, M. Maischberger. A paralle literated tabu search heuristic for vehicle routing problems[J]. Computers and Operations Research, 2012, 39(9): 2033-2050.

[12] F. Shi, H. Wang, L. Yu, F. Hu. Matlab intelligent algorithm 30 case studies[M]. beijing: beihang university press, 2011.

[13] Z. Y. Li. Research on reverse logistics network optimization model of waste electronic products under uncertainty[D]. Beijing Jiaotong University, 2013 